\title{
Situación de los egresados de Administración de Empresas en el mercado laboral: caso UNICATÓLICA*
}

\section{Graduates labor market status: the case of Unicatólica}

Alexandra Hoyos Bravo**,

Rec: Abril 2017

Acep: Octubre 2017

Sully Tatiana Gutiérrez González***

\section{Resumen}

Las diferentes evaluaciones y seguimientos a egresados, contribuyen en gran manera a mejorar los procesos de calidad de las instituciones educativas, facilita la autoevaluación, autocontrol y la capacidad de adaptarse a las necesidades de la sociedad y claramente fortalece la gestión de los centros de egresados. De 2012 a 2015, la Fundación Universitaria Católica Lumen Gentium - Unicatólica, egresó aproximadamente 4.713 personas, de las cuales el $55.32 \%$ son profesionales, y de estos, cerca del 46\% son delprograma de Administración de Empresas. Ante el gran número de egresados y el llamado hacia todas las Instituciones de Educación Superior -IES- para desarrollar procesos académicos con calidad acordes a las necesidades de la sociedad, es preciso analizar la inserción del egresado de Administración de Empresas en el mercado laboral. Este estudio, se enfoca en la caracterización de los graduados de Unicatólica y su inserción laboral, a través de una encuesta y dos grupos focales. Los resultados generales muestran que la mayoría de los graduados son de nivel socioeconómico 3; están empleados, tienen entre 20 y 29 años, eligen su carrera por vocación y consideran que la educación recibida está de acuerdo con sus ocupaciones.

Palabras claves: egresados, relevancia, calidad educativa, mercado laboral.

\footnotetext{
* Este artículo es producto del proyecto de investigación institucional denominado El Egresado Unicatólica y su inserción laboral.

** Magíster en Economía. Universidad Autónoma de Occidente. Docente investigadora Fundación Universitaria Católica Lumen Gentium. Grupo de Investigación Interdisciplinario en Conocimiento Organizacional - GIICO. Correo-e: ahoyosb@unicatolica.edu.co

*** Candidata Magíster en Administración. Universidad Autónoma de Occidente. Docente investigadora Fundación Universitaria Católica Lumen Gentium. Grupo de Investigación Interdisciplinario en Conocimiento Organizacional - GIICO. Correo-e: sgonzalez@unicatolica.edu.co
} 


\section{Abstract}

The different assessment and follow-up of graduates contribute greatly to improve the quality processes of educational institutions, facilitates self-evaluation, self-control and the capacity to adapt to the needs of society and clearly strengthens the management of the graduate's centers. From year 2012 to 2015, the Fundación Universitaria Católica Lumen Gentium - Unicatólica, graduated approximately 4.713 people, of which $55.32 \%$ were professionals, and of these, about $46 \%$ were from the Business Administration program. Given the large number of graduates and the call for all Institutions of Higher Education -IES- to develop academic processes with quality and according to the needs of the society, it is necessary to analyze the insertion of the graduates of Business Administration in the labor market.This study focuses on the characterization of the Unicatólica graduates and their labor market insertion, through a survey and two focus groups.The general results show that the majority of the graduates are of socioeconomic level 3; they are employed, they are between 20 and 29 years old, they chose their career by vocation and consider that the education received is according with their occupations.

Keywords: graduates, relevance, quality in education, labor market.

\section{Introducción}

En el nuevo escenario de globalización, las IES buscan ofrecer una propuesta educativa acorde con las necesidades de la sociedad actual, garantizando pertinencia y calidad en la educación impartida. En este sentido, la introducción de procesos de evaluación del Sistema Nacional de Acreditación (SNA), busca fortalecer la calidad de la Educación Superior, como medio de ajuste y verificación de la calidad y relevancia educativa de los programas de acuerdo a las necesidades y el desarrollo del país; para lo cual evalúa varios factores como la pertinencia educativa y el de impacto de los egresados en el medio, entre otros (Consejo Nacional de Acreditación - CNA, 2013).

Las diferentes evaluaciones y seguimientos a egresados, contribuyen en gran manera a mejorar los procesos de calidad de las instituciones educativas, facilita la autoevaluación, autocontrol y la capacidad de adaptarse a las necesidades de la sociedad y del entorno para impactarlos de manera positiva. Por ende, la información sobre los egresados y su trayectoria profesional y ocupacional es de gran importancia para Unicatólica, dado que le permite conocer el desempeño de sus egresados en la sociedad, y a la vez, si la oferta educativa es coherente y conveniente para contribuir al desarrollo sostenible de la región.

Del año 2012 al 2015, Unicatólica egresó aproximadamente 4.713 personas, de las cuales el $55.32 \%$ son profesionales, el $10.87 \%$ son especialistas, el 32.27\% son tecnólogos y el 1.12\% son técnicos. Los egresados del Programa de Administración de Empresas son
2.188, que corresponden al 46\% de los egresados de los programas propios de la Institución (Departamento de Egresados, Unicatólica en 2016).

A pesar de este número importante de egresados, la Institución no contaba con una investigación acerca de los egresados y su estado ocupacional actual. Es por esta razón que se crea la necesidad por parte de la Consiliatura, máximo ente financiero y administrativo de la Institución, de conocer cómo, a través de los años, los egresados han impactado en su entorno y si las competencias y conocimientos adquiridos en la Institución son los relevantes y necesarios para su desempeño profesional, reconociendo así el proceso educativo que se ha venido adelantando. De igual manera, se busca dilucidar los cambios que sean necesarios en las funciones sustantivas (currículo, investigación y proyección social) que permitan mejorar la formación y el posicionamiento de los egresados.

En este sentido se generó el proyecto El egresado Unicatólica y su inserción laboral, el cual tenía como objetivo principal determinar el impacto del egresado Unicatólica en el mercado laboral. El estudio permitió realizar una caracterización del egresado del Programa de Administración de Empresas, analizar su inserción en el mercado laboral por medio de la definición del perfil ocupacional de este, identificando los requerimientos del medio empresarial para su vinculación laboral.

El proyecto dio como resultado este artículo que permite conocer la situación actual de los egresados del 
programa de Administración de Empresas y la articulación de estos egresados en el medio, de acuerdo a las necesidades del mercado laboral.

\section{Metodología y materiales}

La investigación es un estudio descriptivo enfocado a realizar una caracterización del egresado de Unicatólica, la pertinencia de su formación académica y su inserción laboral.

Se realizó una revisión del estado del arte y de las principales teorías del mercado y la inserción laboral (Damián \& Arellano, 2009; Formichella \& London, 2005; Martínez, 2000), así como sus desequilibrios del mercado laboral (Ángulo, Quejada \&Yánez, 2012; González, 2003; López \& Burgos, 2010) y la pertinencia educativa (Piña, 2006; Unesco, 1995). De igual manera, se llevó a cabo una revisión de estudio sobre la temática de egresados con autores tales como Escobar, Rincón y Osorio (2010); Cortés, Gómez y León (2013) y Arango y Echenique (2014), los cuales analizan el desempeño y la situación de los egresados de Administración de Empresas, logrando indagar sobre el nivel de inserción laboral, la participación de los egresados en las empresas, los sectores económicos en los que laboran, la calidad y pertinencia de la formación, entre otros elementos.

La población objeto son todos los egresados que se encontraban registrados en la base de datos de la institución desde el año 2012 hasta el 2015. De acuerdo con el Departamento de Egresados, eran 4.713 en los programas propios. Por otra parte, fue necesario interactuar con algunos empresarios empleadores de los sectores público y privado, con el fin de conocer su opinión sobre los egresados y sobre las competencias y habilidades primordiales que consideran que estos deben tener para ser vinculados laboralmente.

El estudio se realizó bajo la metodología utilizada por el Observatorio Laboral para la Educación (OLE) del Ministerio de Educación de Colombia (Ministerio de Educación Nacional, 2011), para los estudios de egresados (graduados) de Educación Superior. En este estudio se definen tres momentos, dado que solo se cuenta con una base de egresados con datos fiables desde el 2012, de la siguiente forma:
- Momento 0: egresados 2015

- Momento 1: egresados 2014

- Momento 3: egresados 2012

Con base en la población registrada por el Departamento de Egresados, se procedió a hacer la selección de los egresados de las cohortes y programas definidos para el estudio. Es así como de una población de 2.188 egresados de Administración de Empresas, se pasó a 1.298 egresados que cumplían con los criterios definidos en años de egreso, de los cuales 999 contaban con información de contacto.

Para la distribución inicial del tamaño de la muestra, se realizó el cálculo sobre los egresados iniciales con un nivel de confianza del 95\%, bajo muestreo aleatorio simple, dando como resultado una muestra definitiva para el Programa de Administración de Empresas de 172 encuestas, 64 para los años 2012, 64 para 2015 y 44 para 2014.

El instrumento utilizado fue una encuesta, que contaba con un apartado general y otro específico según el área de formación y/o campo de acción en el mercado laboral. La aplicación del instrumento se hizo de forma presencial, cara a cara y vía telefónica, para los programas propios de la universidad. Para los programas en convenio el cuestionario fue enviado en forma online mediante formulario. El formato de encuesta cuenta con 42 preguntas de la caracterización demográfica y laboral del egresado, el perfil ocupacional y la idoneidad de la formación académica recibida, desde su óptica.

Asimismo, se realizaron dos grupos focales con empresarios de la región, el primero, empresarios o directivos que tenían algún tipo de relacionamiento con la Institución, y el segundo, con empresarios sin ningún vínculo; esto con el fin de conocer de primera mano apreciaciones sobre las competencias, habilidades, desempeño de los egresados y demás factores relevantes de los egresados de los programas.

\section{Resultados}

En este apartado se presentan los resultados de la encuesta realizada a los egresados del Programa de Administración de Empresas, en los años 2012, 2014 y 2015.Se abordará al egresado desde diferentes perspectivas, inician- 
do con una descripción sociodemográfica que permita conocer a los actores principales del estudio, para hacer un análisis sobre la inserción en el mercado laboral y las condiciones laborales. Posteriormente, se estudiará la relación de la formación recibida frente a los requerimientos del medio laboral (pertinencia educativa) y la relación egresado-Unicatólica, para evaluar la percepción y relación del egresado frente a su alma mater. Finalmente, se presentarán las percepciones de los empresarios definidas por los grupos focales realizados.

\section{Algunas características demográficas de los egresados}

El Programa de Administración de Empresas se compone principalmente de egresados en el estrato 3 , seguido del estrato 2 , dentro de los tres momentos evaluados. Este perfil de los egresados en los diferentes momentos (momento 3, 1 y 0) evidencia la población que atiende Unicatólica. La institución desde sus inicios ha buscado impactar de forma particular a las poblaciones con menores ingresos, para lo cual genera oportunidades para acceder a una educación superior de calidad a precios accesibles y lograr, con la formación recibida, mejores condiciones de vida.

En cuestión de sexo, la participación de la mujer en estos escenarios de formación es mayor que la participación de los hombres; más del 50\% de los egresados en el 2012 y 2014 son mujeres, y para el año 2015 la participación de las mujeres ascendió al 65\%. Las edades de estos egresados que participaron en el estudio, están entre los 20 y los 59 años, de los cuales la mayoría se ubica en las edades de 20 a 29 años.

Todas estas características son las que representan los egresados en los años y programa estudiado. En relación a esto, el factor principal por el que decidieron iniciar su proceso de formación y configurarse hoy en día como parte de la comunidad Unicatólica, es la vocación. La mayoría de los egresados referencian que el gusto o el interés por la carrera también es un factor importante para la elección de su enfoque profesional. De igual manera, los egresados encuestados indican otras motivaciones tales como aspectos laborales.

\section{Incorporación al mercado laboral y autoempleo}

Thieme (2007) indica que el nuevo mercado laboral, caracterizado por una alta competitividad, muestra la flexibilidad laboral y con ello la disminución de la estabilidad del empleo, además de crecientes requerimientos educativos, de información y de conocimiento para las distintas ocupaciones; por ende, en este nuevo escenario, la libre movilidad y la integralidad en la formación como emprendedor o empleado es necesaria e importante.

En lo que concierne a la ocupación, en el momento de la encuesta (Figura 1), se encontró que la mayoría de los egresados se desempeña como empleado asalariado, Sin embargo, se observó un decrecimiento de esta actividad y un aumento en el desempleo.

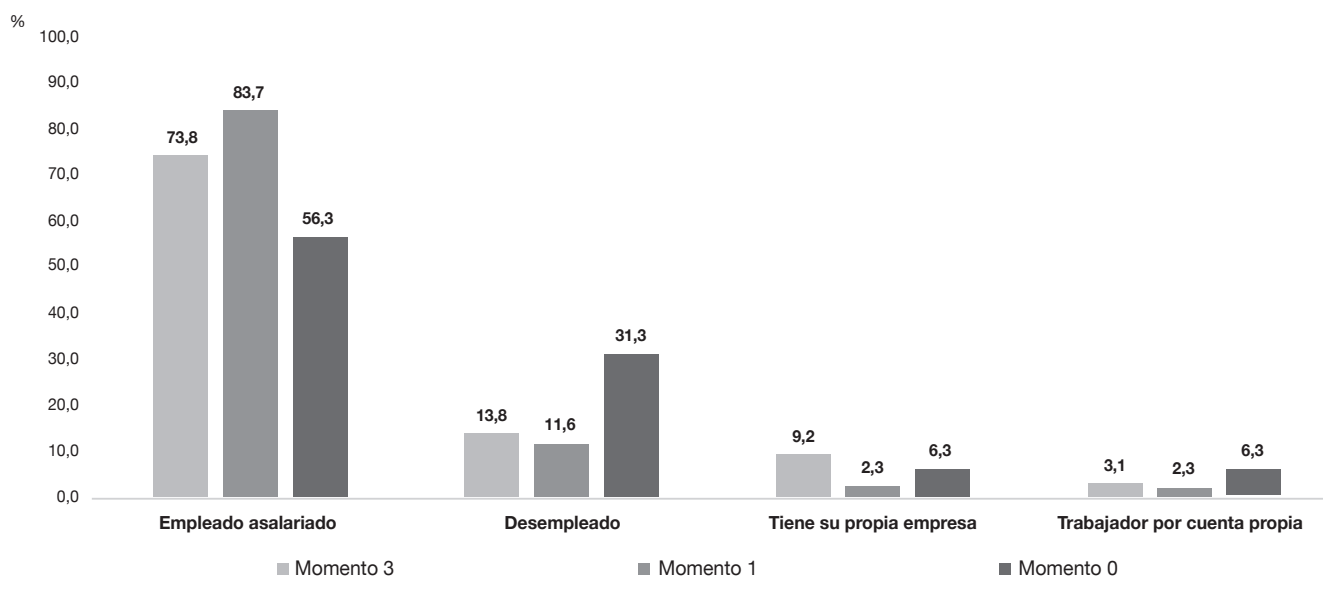

Figura 1. Ocupación principal de los egresados del programa de Administración de Empresas Fuente: Encuesta egresados. Proyecto "El Egresado UNICATÓLICA y su inserción laboral". 2016 
De la misma forma, un porcentaje importante de egresados se encontraba desempleado en el momento de la encuesta, principalmente los egresados del año 2015, con una tasa de desempleo promedio de $19 \%$, mayor en 8.8 puntos a la cifra registrada por la tasa de desempleo para Cali, en el período de análisis.

Por un lado, en cuanto al primer empleo, un porcentaje alto de encuestados indica que su puesto actual no es su primer empleo. Por otro lado, los desempleados son en su mayoría cesantes, es decir, que ya han tenido un empleo anteriormente y se encuentran buscando trabajo, mientras el 14.7\% de los desempleados buscan entrar por primera vez en el mercado laboral.

Además, aunque la tendencia indique que estén aumentando los egresados de Administración de Empresas que entran al mercado laboral, un porcentaje importante lo están haciendo sin experiencia laboral. Esto se evidencia en el aumento en el tiempo de búsqueda de los egresados, dado que mientras que los egresados de los años 2012 y 2014 reportan entre 1 mes hasta 1.5 años sin empleo, los egresados de 2015 indican un período de hasta 3 años. Frente a esta situación, como lo indica Salikof (citado por Miller, 2015), el haber tenido más de un empleo actualmente es una ventaja en el mercado laboral, dado que los empleadores prefieren a los trabajadores que han tenido múltiples experiencias, y algunos de ellos pierden todo interés en candidatos que han pasado demasiado tiempo en un mismo puesto o en la misma empresa, por considerar que probablemente no se vieron en la obligación de mantener un ritmo de cambios como los presentados en el mercado laboral.

Parte de la formación de los egresados es la promoción del espíritu emprendedor, el cual está ligado a la iniciativa y a la acción. Las personas que poseen este espíritu tienen capacidad de innovar, son creativas y flexibles. En el ámbito empresarial, una persona con espíritu empresarial identifica oportunidades de negocio, organiza los recursos para ponerlos en marcha y hacerlos realidad (del Arco \& Blömer, 2012). En este sentido, es importante reconocer estas capacidades en los egresados en los cuales la franja de formación en emprendimiento es de carácter transversal.

Pese a lo definido anteriormente, se puede observar en la Figura 1, que la ocupación de emprendedores y cuenta propia (generadores de empleo y autoempleo), tiene poca participación, ya que solo el 18.7 \% de los administradores afirma haber realizado alguna diligencia para crear empresa.

\section{Rasgos de pertinencia}

El proceso de inserción de un egresado, se remite al momento en que este logra ejercer en un ambiente laboral o empresarial. La mayoría de los egresados de Unicatólica se encuentran ocupados, es decir ya se han insertado en el mundo laboral o empresarial, pero es importante analizar qué tan relacionada esta la ocupación que desarrollan con la formación académica que obtuvieron.

Una vez los egresados están en el ambiente laboral, estos perciben que su ocupación se encuentra muy relacionada con su carrera. En este sentido, consideran que cuentan con la formación para desempeñarse en sus puestos de trabajo.

En cuanto a los diferentes años analizados, los administradores que egresaron en el 2014 superan a los egresados de los otros años, dado que más del $67 \%$ referenciaron que su ocupación está muy relacionada con el cargo que desempeñan en la actualidad, en comparación con un 60\% (2012) y un 56\% (2015) en la misma categoría de respuesta.

$\mathrm{Al}$ analizar las ocupaciones en las que se desempeñan estos egresados, se concluye que estos tienen una mayor participación en actividades o cargos asistenciales, desarrollando actividades de organización, archivo, recepción, auxiliares de diferentes áreas, entre otros.

Respecto al tema de competencias, los administradores de empresas de Unicatólica referencian que, según su experiencia, no se fortaleció la competencia de hablar una segunda lengua, ni el aspecto de prácticas o pasantías universitarias. Un hecho a resaltar es que los egresados de años anteriores perciben en mayor medidala falta de fortalecimiento en la competencia de Inglés como segunda lengua, que los egresados de años más recientes y, por el contrario, frente al tema de prácticas o pasantías laborales, son los egresados de años más recientes los que manifiestan que falta más fortalecimiento en ese aspecto.

En cuestiones de valores diferenciales o ele- 
mentos propios de los egresados de Unicatólica, los administradores de empresas perciben que frente a otros egresados si tienen factores que los diferencian, ya sea a nivel de competencias, valores o habilidades. Frente a estos aspectos, de manera detallada los egresados del 2015 son los que perciben menos factores diferenciales, los egresados para el 2014, los que más perciben elementos diferenciadores como egresados de Unicatólica. En el relacionamiento de la institución con el egresado, la mayoría de los egresados de Administración de Empresas para todos los años de estudio, referenciaron que mantienen alguna relación o contacto con la Institución.

\section{Percepción de los empresarios: exigencias del mercado laboral}

Se realizaron dos grupos focales para conocer las expectativas y opiniones de los empresarios de la región sobre las competencias, conocimientos y valores que les parecen más pertinentes en los profesionales de Administración de Empresas, para que puedan insertarse eficientemente en el mercado laboral. En este sentido, el primer grupo focal se realizó con empresarios que han tenido algún tipo de relación con la Institución, ya sea porque tuvieron estudiantes en práctica o por que han empleado egresados. El segundo grupo, por el contrario, estaba compuesto por empresarios que no tenían ningún tipo de relacionamiento con Unicatólica. En la Tabla 1 se presentan en forma resumida las principales apreciaciones de los dos grupos.

Tabla 1.

Resultados grupos focales con empresarios

\begin{tabular}{|c|c|c|}
\hline Temas & Empresarios relacionados con Unicatólica & Empresarios no relacionados con Unicatólica \\
\hline $\begin{array}{l}\text { Competencias que } \\
\text { cree que debe tener } \\
\text { un administrador de } \\
\text { empresas }\end{array}$ & $\begin{array}{ll}\text { - } & \text { Ofimática } \\
\text { - } & \text { Pensamiento sistémico } \\
\text { - } & \text { Pensamiento crítico } \\
\text { - } & \text { Actitud proactiva } \\
\text { - } & \text { Liderazgo } \\
\text { - } & \text { Comunicación asertiva } \\
\text { - } & \text { Iniciativa } \\
\text { - } & \text { Adaptación al cambio } \\
\text { - } & \text { Relacionamiento con el mundo laboral: pasantías } \\
& \text { y práctica } \\
\text { - } & \text { Servicio }\end{array}$ & $\begin{array}{ll}\text { - } & \text { Comunicación oral y escrita } \\
\text { - } & \text { Creatividad } \\
\text { - } & \text { Pensamiento sistémico } \\
\text { - } & \text { Iniciativa } \\
\text { - } & \text { Concepción ambiental } \\
\text { - } & \text { Ofimática } \\
\text { - } & \text { Manejo de un segundo idioma } \\
\text { - } & \text { Ética, valores, responsabilidad social y ambiental } \\
\text { - } & \text { Trabajo en equipo } \\
\text { - } & \text { Responsabilidad } \\
\text { - } & \text { Capacidad de escucha } \\
\text { - } & \text { Conocimiento del sistema de salud } \\
\text { - } & \text { Programas de calidad } \\
\text { - } & \text { Enfoque global }\end{array}$ \\
\hline $\begin{array}{l}\text { Valores cree que debe } \\
\text { tener un administrador } \\
\text { de empresas }\end{array}$ & $\begin{array}{l}\text { - Los valores deben ser fortalecidos desde la } \\
\text { universidad } \\
\text { - Valores humanos } \\
\text { - } \quad \text { Compromiso y servicio }\end{array}$ & $\begin{array}{ll}\text { - } & \text { Honestidad } \\
\text { - } & \text { Responsabilidad } \\
\text { - } & \text { Excelencia } \\
\text { - } & \text { Manejo del estrés } \\
\text { - } & \text { Conciencia sobre asignaturas de formación humana } \\
\text { - } & \text { Transparencia } \\
\text { - } & \text { Superación/orientación al logro } \\
\text { - } & \text { Felicidad }\end{array}$ \\
\hline $\begin{array}{l}\text { Factores diferenciadores } \\
\text { (generadores de } \\
\text { valor) que tienen los } \\
\text { administradores de } \\
\text { empresas de Unicatólica }\end{array}$ & $\begin{array}{l}\text { - Valores de UNICATÓLICA están definidos, pero no } \\
\text { han sido asumidos por los estudiantes } \\
\text { - No identifican ningún factor diferenciador adicional }\end{array}$ & \\
\hline
\end{tabular}

Fuente: Grupos focales. Proyecto "El Egresado UNICATÓLICA y su inserción laboral". 2016 


\section{Conclusiones}

De acuerdo con los resultados del estudio se puede evidenciar que los egresados del Programa aseguran, en términos generales, estár vinculados en actividades relacionadas con su formación. Sin embargo, los cargos y las ocupaciones realizadas no son las más acordes a su formación.

Asimismo, a pesar de que existe una formación transversal dentro de la carrera en emprendimiento, se observa que muy pocos egresados tiene autoempleo, es decir, son pocos los empresarios y empleados cuenta propia; por ende, se debe incentivar a los estudiantes a la creación de empresas. Para ello se debe fortalecer la Oficina Institucional de Emprendimiento, para contar con el acompañamiento de profesionales especializados que aporten su experiencia para el diseño y la factibilidad del proyecto. En este sentido, para dar sostenibilidad a los emprendedores, es necesario el esfuerzo institucional para conseguir alianzas con entidades financieras o ángeles inversionistas que apalanquen las ideas de negocio.

La relación del programa con el medio empresarial es poca; por ende, se deben fortalecer las prácticas empresariales y visitas a las empresas, para que el estudiante tenga una visión más clara de que puede ser un proceso productivo en la realidad y se acople fácilmente a la vida laboral. Asimismo, es necesario el diálogo constante universidad - empresa con el fin de conocer de primera mano las necesidades y expectativas del empresariado de la región, a la vez que se puedan generar innovaciones y aplicaciones tecnológicas pertinentes.

Además, es importante fortalecer el plan de estudios con la formación de competencias en ofimática, pensamiento sistémico, resolución de problemas, pensamiento crítico, liderazgo y comunicación asertiva, a la vez que se fortalece la formación en valores propia de Unicatólica, que le permita a los egresados contar con una formación integral desde la ética, la responsabilidad social y ambiental.

Igualmente, se hace necesario la implementación de ciclos de capacitación docente para el desarrollo y la aplicación de las nuevas tecnologías de comunicación e información. En este sentido, se deben propender a la internacionalización y globalización del programa, promoviendo además el bilingüismo como parte fundamental del plan de estudios, con el fin de mejorar la competitividad de los egresados.

Unicatólica debe hacer seguimiento y evaluación del desempeño de sus egresados, analizando detalladamente las características del mercado laboral propio de la región, para tener una visión integral, tanto interna como externa, de las implicaciones y realidades que deben enfrentar los futuros profesionales. Por último, todos los ajustes para el fortalecimiento del Programa, deben ir acompañados de una transformación en los procesos administrativos, académicos y financieros institucionales.

\section{Referencias bibliográficas}

Ángulo, G., Quejada, R. \& Yánez, M. (2012). Educación, mercado de trabajo y satisfacción laboral: el problema de las teorías del capital humano y señalización de mercado. Revista de la Educación Superior. 41, 163, 104-120. Recuperado de http://wwww.scielo.org. $m x /$ scielo.php?pid $=$ S0185-27602012000300002Escript $=$ sci arttext

Consejo Nacional de Acreditación - CNA. (2013). Lineamientos para acreditación de programas de pregrado. Recuperado de http://www.cna.gov.co/1741/articles-186359 pregrado 2013.pdf

Damián, J. \& Arellano, LL. (2012). Calidad profesional del técnico superior universitario en administración. Una visión de graduados y de empleadores. Revista Electrónica Actualidades Investigativas en Educación. 9, 2, 1-30.

Del Arco, E. A., \& Blömer, B.V. (2012). Empresa e iniciativa emprendedora. Editorial Paraninfo. Madrid, España.

Formichella, M. M., \& London, S. (2005). Reflexiones acerca de la noción de empleabilidad. Anales de la AAEP.

González, S. M. (2003). Inserción laboral, desajuste educativo y trayectorias laborales de los titulados en formación profesional específica en la isla de Gran Canaria, Las Palmas de Gran Canaria. Universidad de Las Palmas de Gran Canaria, España. Recuperado de http://wwww.eumed. net/tesis-doctorales/smgb/

López, K., \& Burgos, B. (2010). La situación del mercado laboral de profesionista. Revista de la Educación Superior. XXXIX, 19 - 33. 
Martínez, R. M. (2000). Aproximaciones teóricas a los procesos de inserción laboral. Revista del Ministerio de Trabajo e Inmigración. 26, 65-92.

Miller, D. (2015). 48 days to the work you love: preparing for the new normal. B\&H Publishing Group.New York.

Ministerio de Educación Nacional de Colombia. (2011). Observatorio Laboral para la Educación: Seguimiento a los Graduados de la Educación Superior en los últimos 10 años. Ministerio de Educación Nacional de Colombia. Bogotá D.C.
Piña, I. (2006). La universidad en el siglo XXI. Revista de la Educación Superior. XXXV, 115 - 122.

Thieme, C. (2007). El desarrollo de competencias de empleabilidad en dos universidades chilenas". Un estudio empírico. OIKOS. 47-72.

UNESCO. (1995). Documento para el cambio y el desarrollo de la educación superior. En: Disyuntiva actual de la educación superior: documentos (pp. 63 - 74). Praxis - UNAM. México. 